\title{
BIDUALITY IN SPACES OF HOLOMORPHIC FUNCTIONS
}

\author{
P. GALINDO ${ }^{1}$, M. MAESTRE ${ }^{2}$ AND P. RUEDA
}

\begin{abstract}
This paper contains characterizations of the bidual space of some closed subspaces of $\mathcal{H}_{b}(U)$, the space of holomorphic functions of bounded type defined on an open subset $U$ of a Banach space $X$, where $U$ is either a bounded balanced open set or the whole space $X$.
\end{abstract}

In recent years several authors have dealt with the matter of describing the bidual of some given spaces of polynomials or holomorphic functions. See, for instance [1], [4], [7], [8], [13], [14] and [15]. Prieto (Theorem 12 of [13]) states that $\mathcal{H}_{b}(X)$ is isomorphic to the bidual space of $\mathcal{H}_{w u}(X)$, the space of holomorphic functions of bounded type which are weakly uniformly continuous on bounded subsets of $X$, if and only if the space of continuous $m$ homogeneous polynomials $\mathcal{P}\left({ }^{m} X\right)$ is isomorphic to the bidual space of $\mathcal{P}_{w u}\left({ }^{m} X\right)=\mathcal{P}\left({ }^{m} X\right) \cap \mathcal{H}_{w u}(X), \forall m \in \mathrm{N}$. According to her we have the following situation: $\mathcal{P}\left({ }^{n} X\right)=\mathcal{P}_{w u}\left({ }^{n} X\right)^{* *}$ for every $n \geq 0$ and since these families are Schauder decompositions of $\mathcal{H}_{b}(X)$ and $\mathcal{H}_{w u}(X)^{* *}$ respectively, apparently both of these spaces coincide algebraically and, hence, topologically. However, $\mathcal{H}(\mathrm{C})$ and $\mathcal{H}(\Delta)$, where $\Delta$ is the open unit ball of $\mathrm{C}$, have the same Schauder decomposition $\left(\mathcal{P}\left({ }^{n} \mathrm{C}\right)\right)_{n}$ but they are not topologically isomorphic (see the remark after Corollary 10.6.12 of [9] or Remark 5). This example shows that given two spaces with Schauder decomposition, to have a topological isomorphism between them we have to assume a stronger condition than mere isomorphisms between the spaces forming the decomposition. In order to clarify this situation we isolate a subclass of Schauder decompositions: the $R$-Schauder decompositions. Concerning that, our main result is Theorem 9.

Given a Banach space $X$ and its dual space $X^{*}$, we denote by $\mathcal{P}_{w^{*}}\left({ }^{m} X^{*}\right)$ $\left(\mathcal{H}_{w^{*}}\left(X^{*}\right)\right.$ respectively) the space of all continuous $m$-homogeneous poly-

\footnotetext{
1 Partially supported by DGICYT(Spain) pr. 91-0326.

2 Partially supported by DGICYT(Spain) pr. 91-0326 and pr. 91-0538.

Received November 11, 1996; in revised form June 4, 1997.
} 
nomials on $X^{*}$ (of all entire functions on $X^{*}$ respectively) which are weak*uniformly continuous on bounded subsets of $X^{*}$.

Valdivia in [14] proves that the bidual space of $\mathcal{P}_{w^{*}}\left({ }^{m} X^{*}\right)$ is isometric to its $\tau_{0}$-closure in $\mathcal{P}\left({ }^{m} X^{*}\right)$ assuming that $\mathcal{P}_{w^{*}}\left({ }^{m} X^{*}\right)$ does not contain copies of $\ell^{1}$, whereas in [15], he proves the analogue result with $\mathcal{H}_{w^{*}}\left(X^{*}\right)$. In particular, he obtains that if $X$ is a Banach space such that $X^{*}$ has the approximation property and $\mathcal{H}_{w^{*}}\left(X^{*}\right)$ contains no copy of $\ell^{1}$, then the bidual space of $\mathcal{H}_{w^{*}}\left(X^{*}\right)$ is canonically identified with $\mathcal{H}_{b}\left(X^{*}\right)$. By using his results for polynomials and the decomposition we introduce, we obtain a new proof of these results and extend them to holomorphic mappings on the unit ball.

Most of the notation is standard. We refer to [10] and [6] for definitions and properties of locally convex spaces and holomorphic functions on infinite dimensional spaces respectively. For an arbitrary locally convex space $E, E^{*}$ denotes the strong dual of $E$. In the sequel we use the notation $(X,\|\|$.$) for an arbitrary complex Banach space, B$ for the open unit ball of $X$ and $U$ for an arbitrary balanced open subset of $X$. $\|\cdot\|^{*}$ means the dual norm in $X^{*}$. We denote by $\mathcal{P}\left({ }^{m} X\right)$ the space of all continuous $m$-homogeneous polynomials on $X$, and by $\mathcal{P}_{w u}\left({ }^{m} X\right)$ the subspace of $\mathcal{P}\left({ }^{m} X\right)$ whose polynomials are weakly uniformly continuous on bounded subsets of $X$. $\mathcal{H}_{b}(U)$ denotes the space of all holomorphic functions of bounded type on $U$, that is, the space of all holomorphic functions on $U$ which are bounded on all $U$-bounded sets. We recall that the $U$-bounded sets are in the case $U=X$ the bounded subsets of $X$, whereas, in the case of an arbitrary open set $U$, they are the bounded subsets of $U$ whose distance to the boundary of $U$ is greater than zero. If $A$ is a $U$-bounded set, we put $\|f\|_{A}:=\sup _{x \in A}|f(x)|$, $f \in \mathcal{H}_{b}(U) . \mathcal{H}_{b}(U)$ will be endowed with the topology $\tau_{b}$ defined by the seminorms $\|.\|_{A}$, where $A$ runs over all $U$-bounded sets. It is well known that $\left(\mathcal{H}_{b}(U), \tau_{b}\right)$ is a Fréchet space. If $f \in \mathcal{H}_{b}(U), \sum_{m=0}^{\infty} P_{m}(f)$ denotes the Taylor series of $f$ at the origin. Let $\mathcal{H}_{w u}(U)$ denote the subspace of $\mathcal{H}_{b}(U)$ of all holomorphic functions on $U$ which are weakly uniformly continuous on all $U$-bounded sets. If $G$ is an open subset of $X^{*}, \mathcal{H}_{w^{*}}(G)$ is the subspace of $\mathcal{H}_{b}(G)$ of all holomorphic functions on $G$ which are weak*-uniformly continuous on all $G$-bounded sets.

Let us recall that a sequence of subspaces $\left(F_{n},\|\cdot\|_{n}\right)_{n}$ of a locally convex space $F$ is a Schauder decomposition of $F$ if each $x \in F$ can be written in a unique way as $\sum_{n=0}^{\infty} x_{n}$ where $x_{n} \in F_{n}$ and the projections $u_{m}$ : $\sum_{n=0}^{\infty} x_{n} \in F \rightarrow \sum_{n=0}^{m} x_{n} \in F$ are all continuous.

Given a sequence of Banach spaces $\left(E_{n},\|\cdot\|_{n}\right)_{n}$ and $0<R \leq \infty$ the Köthe sequence space $\lambda^{1}\left(A_{R} ;\left(E_{n}\right)_{n}\right)$ (where $A_{R}=\left\{\left(r^{n}\right)_{n}: 0<r<R\right\}$ ) is the Fréchet space $\left\{\left(x_{n}\right)_{n} \in \prod_{n} E_{n}: p_{r}\left(\left(x_{n}\right)_{n}\right)=\sum_{n=0}^{\infty}\left\|x_{n}\right\|_{n} r^{n}<\infty \forall r, 0<r<R\right\}$, endowed with the topology given by the seminorms $\left\{p_{r}\right\}_{0<r<R}$. When $E_{n}=\mathrm{C}$ for every 
$n=0,1, \ldots$, this Köthe sequence space is denoted by $\lambda^{1}\left(A_{R}\right)$. By CauchyHadamard's formula $\lambda^{1}\left(A_{R} ;\left(E_{n}\right)_{n}\right)=\left\{\left(x_{n}\right)_{n} \in \prod_{n} E_{n}: \limsup _{n}\left\|x_{n}\right\|_{n}^{1 / n} \leq \frac{1}{R}\right\}$. One can readily check that for $0<R<\infty, \lambda^{1}\left(A_{R} ;\left(E_{n}\right)_{n}\right)=\Lambda_{1}^{1}\left((m)_{m \in \mathrm{N}} ;\left(E_{n}\right)_{n}\right)$ a finite type power series space and for $R=\infty, \lambda^{1}\left(A_{\infty} ;\left(E_{n}\right)_{n}\right)=$ $\Lambda_{\infty}^{1}\left((m)_{m \in \mathrm{N}} ;\left(E_{n}\right)_{n}\right)$ an infinite type power series space (see [9] p. 211). Under the canonical identification $x \in E_{n} \leadsto(0, \ldots, 0, x, 0, \ldots) \in \lambda^{1}\left(A_{R} ;\left(E_{n}\right)_{n}\right)$, one can check that $\left(E_{n}\right)_{n}$ is a Schauder decomposition of $\lambda^{1}\left(A_{R} ;\left(E_{n}\right)_{n}\right)$.

In our process to obtain results on biduals of some spaces of holomorphic functions we have isolated certain properties of their natural, i.e. polynomial, Schauder decomposition, which hold for their biduals as well. These spaces turned out to be power series spaces of the above type and only to emphasize the role played by such decompositions, we will label $R$-Schauder to any decomposition of a Fréchet space $E$ satisfying condition $(R)$ stated in the next theorem, which gives a characterization of those power series spaces that will be useful in the infinite dimensional holomorphy.

THEOREM 1. Given $\left(E_{n},\|\cdot\|_{n}\right)_{n}, \lambda^{1}\left(A_{R} ;\left(E_{n}\right)_{n}\right)$ is topologically isomorphic to any Fréchet space $E$ that has $\left(E_{n}\right)_{n}$ as a Schauder decomposition and satisfies the following condition: (R) for every sequence $\left(x_{n}\right)_{n}, x_{n} \in E_{n}$, the series $\sum_{n=0}^{\infty} x_{n}$ converges in $E$ if and only if $\lim \sup _{n}\left\|x_{n}\right\|_{n}^{1 / n} \leq \frac{1}{R}$.

Proof. Let $\phi: E \longrightarrow \lambda^{1}\left(A_{R} ;\left(E_{n}\right)_{n}\right)$ defined by $\phi\left(\sum_{n=0}^{\infty} x_{n}\right)=\left(x_{n}\right)_{n}$ for all $x=\sum_{n=0}^{\infty} x_{n} \in E$.

Since for any sequence of non negative real numbers $\left(\alpha_{n}\right)_{n}$ and any $0<R \leq \infty$ the condition $\limsup _{n} \alpha_{n}^{1 / n} \leq 1 / R$ is equivalent to $\sum_{n=0}^{\infty} r^{n} \alpha_{n}<\infty$ $\forall r: 0<r<R$, the map $\phi$ is an algebraic isomorphism. We prove that $\phi$ is also a topological isomorphism. Indeed, for every $0<r<R$ the set $\left\{x \in E: p_{r}(\phi(x)) \leq 1\right\}=\bigcap_{l=0}^{\infty}\left\{x=\sum_{m=0}^{\infty} x_{m} \in E: \sum_{m=0}^{l} r^{m}\left\|x_{m}\right\|_{m} \leq 1\right\}$ is a barrel in the Fréchet space $E$, hence a neighbourhood of zero. Thus $\phi$ is continuous and therefore, as a consequence of the open mapping theorem, $\phi$ will be a topological isomorphism.

In Examples 2 and 4 below it is used that $\left(\mathcal{P}\left({ }^{m} X\right)\right)_{m}\left(\left(\mathcal{P}_{w u}\left({ }^{m} X\right)\right)_{m}\right)$ is a Schauder decomposition of $\mathcal{H}_{b}(U)$ [13] $\left(\mathcal{H}_{w u}(U)\right.$ [2], [13] respectively). If $f \in \mathcal{H}_{w u}(X)$, Aron proved in Proposition 1.5b of [2] that $P_{m}(f) \in \mathcal{P}_{w u}\left({ }^{m} X\right)$ by using Cauchy's integral formula. It is well known that the same argument also works in the case of non entire functions and it can also be used to prove that $\left(\mathcal{P}_{w^{*}}\left({ }^{m} X^{*}\right)\right)_{m}$ is a Schauder decomposition of $\mathcal{H}_{w^{*}}(G)$ when $G$ is a balanced open set.

ExAmples 2. By using Cauchy inequalities we obtain that

a) The family $\left(\mathcal{P}\left({ }^{m} X\right),\|\cdot\|_{B}\right)_{m}$ is an $\infty$-Schauder decomposition of $\mathcal{H}_{b}(X)$.

b) The family $\left(\mathcal{P}\left({ }^{m} X\right),\|.\|_{B}\right)_{m}$ is an $R$-Schauder decomposition of $\mathcal{H}_{b}(R B)$. 
c) Let $U \subset X$ be a bounded balanced open set. The family $\left(\mathcal{P}\left({ }^{m} X\right),\|\cdot\|_{U}\right)$ is a 1 -Schauder decomposition of $\mathcal{H}_{b}(U)$.

REMARK 3. If $\left(E_{n},\|\cdot\|_{n}\right)_{n}$ is an $R$-Schauder decomposition of the Fréchet space $E$ and $F$ is a closed subspace of $E$ so that $\left(F \cap E_{n},\|.\|_{n}\right)_{n}$ is a Schauder decomposition of $F$, then $\left(F \cap E_{n},\|\cdot\|_{n}\right)_{n}$ is an $R$-Schauder decomposition of $F$.

As a consequence of Remark 3 and Examples 2 we obtain:

EXAmples 4. a') $\left(\mathcal{P}_{w u}\left({ }^{m} X\right),\|\cdot\|_{B}\right)_{m}$ is an $\infty$-Schauder decomposition of $\mathcal{H}_{w u}(X)$.

b') $\left(\mathcal{P}_{w u}\left({ }^{m} X\right),\|.\|_{B}\right)_{n}$ is an $R$-Schauder decomposition of $\mathcal{H}_{w u}(R B)$.

c') Let $U \subset X$ be a bounded balanced open set. The family $\left(\mathcal{P}_{w u}\left({ }^{m} X\right),\|\|_{U}\right)$ is a 1-Schauder decomposition of $\mathcal{H}_{w u}(U)$.

a") Let $B^{*}$ be the open unit ball of $X^{*}$. The family $\left(\mathcal{P}_{w^{*}}\left({ }^{m} X^{*}\right),\|\cdot\|_{B^{*}}\right)_{m}$ is an $\infty$-Schauder decomposition of $\mathcal{H}_{w^{*}}\left(X^{*}\right)$.

b") Let $B^{*}$ be the open unit ball of $X^{*}$ and let $R>0$. The family $\left(\mathcal{P}_{w^{*}}\left({ }^{m} X^{*}\right),\|.\|_{B^{*}}\right)_{m}$ is an $R$-Schauder decomposition of $\mathcal{H}_{w^{*}}\left(R B^{*}\right)$.

c") Let $G$ be a bounded balanced open subset of $X^{*}$. The family $\left(\mathcal{P}_{w^{*}}\left({ }^{m} X^{*}\right),\|\cdot\|_{G}\right)_{m}$ is a 1-Schauder decomposition of $\mathcal{H}_{w^{*}}(G)$.

REMARK 5. Given $0<R<\infty$ one can easily check that $\lambda^{1}\left(A_{R},\left(E_{n}\right)_{n}\right)$ is topologically isomorphic to $\lambda^{1}\left(A_{1},\left(E_{n}\right)_{n}\right)$. Therefore a natural question arises: is it possible to establish a topological isomorphism between two Fréchet spaces having one an $R$-Schauder decomposition, $0<R<\infty$, and the other one an $\infty$-Schauder decomposition? Since we have defined $R$ Schauder decompositions having in mind the spaces of holomorphic functions of bounded type the question could be stated as: is it possible to find a Banach space $X$ such that $\mathcal{H}_{b}(X)$ is topologically isomorphic to $\mathcal{H}_{b}(B)$ ? (We have pointed out above that this is not true when $X=\mathrm{C}$ ).

The answer to both questions is negative and has been given to us by José Bonet in a personal communication which we gratefully acknowledge and where he pointed out the power series approach to the $R$-Schauder decompositions. This is the way he proceeds:

A Fréchet space $E$ is said to have property (DN) if given a fundamental system of seminorms $\left(\|\cdot\|_{n}\right)_{n \in \mathrm{N}}$ there is a continuous norm $\|\cdot\|$ on $E$ such that for each $n \in \mathrm{N}$ there exists $m \in \mathrm{N}$ satisfying $\|x\|_{n} \leq s\|x\|+s^{-1}\|x\|_{m} \forall s>0$ $\forall x \in E$. Property (DN) is preserved under topological isomorphisms. The spaces $\lambda^{1}\left(A_{R}\right)$ have not property (DN) when $0<R<\infty$ and $\lambda^{1}\left(A_{\infty}\right)$ has it (see Theorem 21.7.5 of [9]). Moreover it is not difficult to check that for any sequence of Banach spaces $\left(E_{n}\right)_{n}$ and any $0<R \leq \infty$ the space $\lambda^{1}\left(A_{R},\left(E_{n}\right)_{n}\right)$ 
has property (DN) if and only if $\lambda^{1}\left(A_{R}\right)$ has it. Thus $\lambda^{1}\left(A_{\infty},\left(E_{n}\right)_{n}\right)$ is not topologically isomorphic to $\lambda^{1}\left(A_{R},\left(E_{n}\right)_{n}\right)$ for any $0<R<\infty$. Therefore, given a Banach space $X$, the space $\mathcal{H}_{b}(X)\left(\mathcal{H}_{w u}(X), \mathcal{H}_{w^{*}}\left(X^{*}\right)\right)$ is not topologically isomorphic to $\mathcal{H}_{b}(B)\left(\mathcal{H}_{w u}(B), \mathcal{H}_{w^{*}}\left(B^{*}\right)\right.$ respectively).

Our next aim is to study when a topological isomorphism occurs between spaces $E$ and $F$ having $R$-Schauder decompositions of the same type. Although Theorem 8 below also follows from the power series spaces techniques, we confine ourselves to the context of $R$-Schauder decompositions since it is completely natural to the holomorphy and still keeps the paper self-contained without enlarging it too much.

Lemma 6. If $\left(E_{n}\right)_{n}$ is an $R$-Schauder decomposition of $E, 0<R \leq \infty$, then $\left(E_{n}\right)_{n}$ is an $\mathcal{S}$-absolute decomposition of $E$.

Proof. Since $E$ is a Fréchet space, we just have to prove that $\left(E_{n}\right)_{n}$ is an $\mathcal{S}$-Schauder decomposition of $E$ (Proposition 3.10 of [6]).

Let $\left(a_{n}\right)_{n} \in \mathcal{S}:=\left\{\left(a_{n}\right)_{n} \subset \mathrm{C}: \lim \sup _{n}\left|a_{n}\right|^{1 / n} \leq 1\right\}$ and let $x=\sum_{m=0}^{\infty} x_{m} \in E$. We have to prove that $\sum_{m=0}^{\infty} a_{n} x_{n} \in E$. Since

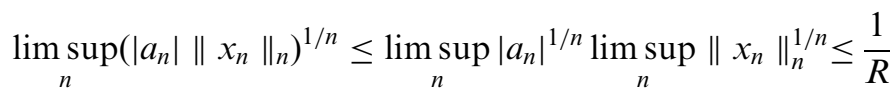

it follows from the definition of $R$-Schauder decomposition that $\sum_{m=0}^{\infty} a_{n} x_{n} \in E$.

For $0<r<R$ we set $\mathcal{B}_{r}:=\left\{x \in E: p_{r}(x) \leq 1\right\}$. By Theorem 1 , the family $\left\{\frac{1}{s} \mathcal{B}_{r}\right\}_{s>0,0<r<R}$ is a fundamental system of neighbourhoods of zero. Let $\mathcal{B}_{r}^{\circ}$ be the polar of $\mathcal{B}_{r}$ in $E^{*}$ and let $\mathcal{B}_{r}^{\circ \circ}$ be the polar of $\mathcal{B}_{r}^{\circ}$ in $E^{* *}$. Since $E^{* *}$ is a Fréchet space, the family $\left\{\frac{1}{s} \mathcal{B}_{r}^{\circ}\right\}_{s>0,0<r<R}$ is a fundamental system of neighbourhoods of zero in it.

Lemma 7. i) Let $\phi_{m} \in E_{m}^{*}$. Then $\phi_{m} \in \mathcal{B}_{r}^{\circ}$ if and only if $\left\|\phi_{m}\right\|_{m}^{*} \leq r^{m}$.

ii) If $\phi=\sum_{n=0}^{\infty} \phi_{n} \in \mathcal{B}_{r}^{\circ}$ then $\left\|\phi_{m}\right\|_{m}^{*} \leq r^{m}, \forall m \in \mathrm{N}$.

Proof. i) Assume that $\phi_{m} \in \mathcal{B}_{r}^{\circ}$, then

$\left\|\phi_{m}\right\|_{m}^{*}=\sup _{x_{m} \in E_{m},\left\|x_{m}\right\|_{m} \leq 1}\left|\phi_{m}\left(x_{m}\right)\right|=\sup _{x_{m} \in r^{m} \mathcal{B}_{r} \cap E_{m}}\left|\phi_{m}\left(x_{m}\right)\right|=r^{m} \sup _{x_{m} \in \mathcal{B}_{r} \cap E_{m}}\left|\phi_{m}\left(x_{m}\right)\right| \leq r^{m}$

for all $m \in \mathrm{N}$.

Conversely, if $\phi_{m} \in E_{m}^{*}$ is such that $\left\|\phi_{m}\right\|_{m}^{*} \leq r^{m}$, then given $x \in \mathcal{B}_{r}$, $x=\sum_{n=0}^{\infty} x_{n}$

$$
\left|\phi_{m}(x)\right|=\left|\phi_{m}\left(x_{m}\right)\right| \leq \frac{1}{r^{m}}\left\|\phi_{m}\right\|_{m}^{*} \leq 1 .
$$

ii) this follows from (i) and the definitions of $\mathcal{B}_{r}$ and $p_{r}$. 
THEOREM 8. If $\left(E_{n},\|\cdot\|_{n}\right)_{n}$ is an R-Schauder decomposition of $E$, $0<R \leq \infty$, then $\left(E_{n}^{* *},\|.\|_{n}^{* *}\right)_{n}$ is an $R$-Schauder decomposition of $E^{* *}$.

Proof. By Lemma $6,\left(E_{n},\|\cdot\|_{n}\right)_{n}$ is an $\mathcal{S}$-absolute decomposition of $E$. Therefore $\left(E_{n}^{* *},\|\cdot\|_{n}^{* *}\right)_{n}$ is an $\mathcal{S}$-absolute decomposition of $E^{* *}$ (Proposition 3.11 of [6]). Given $0<r<R, r<s<R$ and $G=\sum_{m=0}^{\infty} G_{m} \in E^{* *}$, with $G_{m} \in E_{m}^{* *}$, there exists $M>0$ so that $G \in M \mathcal{B}_{s}^{\circ \circ}$. By Lemma 7.i) $\phi_{m} \in E_{m}^{*}$ satisfies $\left\|\phi_{m}\right\|_{m}^{*} \leq 1$ if and only if $s^{m} \phi_{m} \in \mathcal{B}_{s}^{\circ} \cap E_{m}^{*}$. Hence,

$$
\begin{aligned}
r^{m}\left\|G_{m}\right\|_{m}^{* *} & =r^{m} \sup _{\left\|\phi_{m}\right\|_{m}^{*} \leq 1}\left|G_{m}\left(\phi_{m}\right)\right| \\
& =M\left(\frac{r}{S}\right)^{m} \sup _{s^{m} \phi_{m} \in \mathcal{B}_{s}^{\circ} \cap E_{m}^{*}}\left|\frac{G\left(s^{m} \phi_{m}\right)}{M}\right| \leq M\left(\frac{r}{S}\right)^{m} \sup _{\phi \in \mathcal{B}_{s}^{\circ}}\left|\frac{G(\phi)}{M}\right| \leq M\left(\frac{r}{s}\right)^{m} .
\end{aligned}
$$

Therefore the series $\sum_{m=0}^{\infty} r^{m}\left\|G_{m}\right\|_{m}^{* *}$ converges for all $0<r<R$, and by the Cauchy-Hadamard formula, $\lim \sup _{m}\left(\left\|G_{m}\right\|_{m}^{* *}\right)^{1 / m} \leq \frac{1}{R}$.

Now suppose $\lim \sup _{m}\left(\left\|G_{m}\right\|_{m}^{* *}\right)^{1 / m} \leq \frac{1}{R}$. We have to show that the series $\sum_{m=0}^{\infty} G_{m} \beta\left(E^{* *}, E^{*}\right)$-converges. Since the family $\left\{s \mathcal{B}_{r}^{\circ}\right\}_{s>0,0<r<R}$ forms a fundamental system of $\beta\left(E^{*}, E\right)$-bounded sets, it is enough to prove that $\sum_{m=0}^{\infty} G_{m}$ converges uniformly on $s \mathcal{B}_{r}^{\circ}$, for all $s>0$ and $0<r<R$. By Lemma 7.ii) if $\phi=\sum_{m=0}^{\infty} \phi_{m} \in s \mathcal{B}_{r}^{\circ}$ then $\left\|\phi_{m}\right\|_{m}^{*} \leq s r^{m}, \forall m \in \mathrm{N}$. Hence $\left|G_{m}(\phi)\right|=\left|G_{m}\left(\phi_{m}\right)\right| \leq\left\|G_{m}\right\|_{m}^{* *}\left\|\phi_{m}\right\|_{m}^{*} \leq s r\left\|G_{m}\right\|_{m}^{* *}, \forall m \in \mathrm{N}$. Thus $\sum_{m=0}^{\infty} G_{m}$ is uniformly Cauchy on $s \mathcal{B}_{r}^{\circ}$, for $0<r<R$ and $s>0$. Since $E^{* *}$ is a Fréchet space there exists $G:=\sum_{m=0}^{\infty} G_{m} \in E^{* *}$.

THEOREM 9. Let $\left(E_{n},\|\cdot\|_{n}\right)_{n}$ and $\left(F_{n},\|\cdot\|_{n}\right)_{n}$ be R-Schauder decompositions of the Fréchet spaces $E$ and $F$ respectively $(0<R \leq \infty)$.

Assume that there exist algebraic isomorphisms $T_{m}: E_{m} \longrightarrow F_{m} \forall m \in \mathrm{N}$ so that:

i) In case $0<R<\infty$, (Condition I) for each $t>1$ there exist $a_{t}, b_{t}>0$ such that

$$
\left\|T_{m}\left(x_{m}\right)\right\|_{m} \leq a_{t} t^{m}\left\|x_{m}\right\|_{m} \text { and }\left\|x_{m}\right\|_{m} \leq b_{t} t^{m}\left\|T_{m}\left(x_{m}\right)\right\|_{m} \forall x_{m} \in E_{m}, \quad \forall m \in \mathrm{N} \text {. }
$$

ii) In case $R=\infty$, (Condition II) there exist $t, t^{\prime}>0$ and $a_{t}, b_{t^{\prime}}>0$ such that $\left\|T_{m}\left(x_{m}\right)\right\|_{m} \leq a_{t} t^{m}\left\|x_{m}\right\|_{m}$ and $\left\|x_{m}\right\|_{m} \leq b_{t^{\prime}}\left(t^{\prime}\right)^{m}\left\|T_{m}\left(x_{m}\right)\right\|_{m} \forall x_{m} \in E_{m}, \forall m \in \mathrm{N}$.

Then the map $T: x=\sum_{m=0}^{\infty} x_{m} \in E \longrightarrow T(x):=\sum_{m=0}^{\infty} T_{m}\left(x_{m}\right) \in F$ is a topological isomorphism.

Conversely, if there exists a topological isomorphism $T: E \longrightarrow F$ so that $T\left(E_{m}\right) \subset F_{m}, \forall m \in \mathrm{N}$, then $T\left(E_{m}\right)=F_{m}$ and $T_{m}:=\left.T\right|_{E_{m}}$ are topological isomorphisms satisfying Condition I in case $0<R<\infty$ and Condition II in case $R=\infty$. 
Proof. To prove that $T$ is well defined we have to show that $\sum_{m=0}^{\infty} r^{m}\left\|T_{m}\left(x_{m}\right)\right\|_{m}$ converges for $0<r<R$.

Suppose $0<R<\infty$. Let $r<s<R$. By Condition I there exists $a>0$ so that $\left\|T_{m}\left(x_{m}\right)\right\|_{m} \leq a\left(\frac{\Im}{r}\right)^{m}\left\|x_{m}\right\|_{m}, \quad m \in \mathrm{N}$. Hence

$$
r^{m}\left\|T_{m}\left(x_{m}\right)\right\|_{m} \leq a s^{m}\left\|x_{m}\right\|_{m}, \quad m \in \mathrm{N} .
$$

Now suppose $R=\infty$. Then by Condition II there exist $s, a>0$ so that $\left\|T_{m}\left(x_{m}\right)\right\|_{m} \leq a s^{m}\left\|x_{m}\right\|_{m}, \quad m \in \mathrm{N}$. Hence

$$
r^{m}\left\|T_{m}\left(x_{m}\right)\right\|_{m} \leq a(s r)^{m}\left\|x_{m}\right\|_{m}, \quad m \in \mathrm{N} .
$$

Thus we obtain convergence in both cases.

Clearly, $T$ is linear. By Theorem 1 the family $\left\{q_{r}\right\}_{0<r<R}$, where $q_{r}(y):=\sum_{m=0}^{\infty} r^{m}\left\|y_{m}\right\|_{m}, y=\sum_{m=0}^{\infty} y_{m} \in F$, is a fundamental system of continuous seminorms on $F$.

If $0<R<\infty$, then it follows from (1) that $T\left(\left\{x \in E: p_{s}(x) \leq \frac{1}{a}\right\}\right)$ $\subset\left\{y \in F: \quad q_{r}(y) \leq 1\right\}$. If $R=\infty$, then it follows from (2) that $T\left(\left\{x \in E: p_{r s}(x) \leq \frac{1}{a}\right\}\right) \subset\left\{y \in F: q_{r}(y) \leq 1\right\}$. These inclusions prove in both cases the continuity of $T$.

Let $V$ be a map defined from $F$ into $E$ as $V\left(\sum_{m=0}^{\infty} y_{m}\right)=\sum_{m=0}^{\infty} T_{m}^{-1}\left(y_{m}\right)$ for every $y=\sum_{m=0}^{\infty} y_{m} \in F$. In an analogous way to that of $T$ it can be proved that $V$ is well defined and continuous. One can easily check that $V$ is the inverse map of $T$.

Let us now show the converse statement. Define $T_{m}:=\left.T\right|_{E_{m}}: E_{m} \longrightarrow F_{m}$ for every $m \in \mathrm{N}$. $T_{m}$ is a one-to-one linear mapping.

We now prove that each $T_{m}$ is onto. Let $y_{m} \in F_{m} \subset F$. Since $T$ is onto, there exists $x=\sum_{n=0}^{\infty} x_{n} \in E$ such that $T(x)=y_{m}$. As $T$ is continuous and linear $y_{m}=T(x)=\sum_{n=0}^{\infty} T\left(x_{n}\right)=\sum_{n=0}^{\infty} T_{n}\left(x_{n}\right)$ and using the uniqueness of the above sum it follows that $T_{n}\left(x_{n}\right)=0$ for every $n \neq m$ and $y_{m}=T_{m}\left(x_{m}\right)$ where $x_{m} \in E_{m}$. Hence $T_{m}\left(E_{m}\right)=F_{m}$.

Finally we check that Conditions I and II are satisfied. Let $0<r<R$. Since $T$ is continuous there exists $0<s<R$ (we may suppose without loss of generality that $s>r)$ and there exists $a>0$ such that $T\left(\left\{x \in E: p_{s}(x) \leq a\right\}\right)$ $\subset\left\{y \in F: q_{r}(y) \leq 1\right\}$. Hence $q_{r}(T(x)) \leq \frac{1}{a} p_{s}(x) \quad \forall x \in E$. In particular, if $x_{m} \in E_{m}$, then $q_{r}\left(T\left(x_{m}\right)\right) \leq \frac{1}{a} p_{s}\left(x_{m}\right), \quad$ or equivalently, $\quad r^{m}\left\|T_{m}\left(x_{m}\right)\right\|_{m} \leq$ $\frac{1}{a} s^{m}\left\|x_{m}\right\|_{m}$. Hence $\left\|T_{m}\left(x_{m}\right)\right\|_{m} \leq \frac{1}{a}\left(\frac{s}{r}\right)^{m}\left\|x_{m}\right\|_{m}$.

i) Suppose $0<R<\infty$. If $r$ tends to $R^{-}$, then $s$ tends to $R^{-}$, hence $\frac{s}{r}$ tends to $1^{+}$. Thus, given $t>1$ there exists $a_{t}>0$ so that $\left\|T_{m}\left(x_{m}\right)\right\|_{m} \leq a_{t} t^{m}\left\|x_{m}\right\|_{m}$ $\forall x_{m} \in E_{m}$.

ii) If $R=\infty$, then there exist $t>0$ and $a_{t}>0$ so that $\left\|T_{m}\left(x_{m}\right)\right\|_{m} \leq$ $a_{t} t^{m}\left\|x_{m}\right\|_{m} \quad \forall x_{m} \in E_{m} \quad \forall m \in \mathrm{N}$.

Finally, since $T$ is open there exist $b>0$ and $0<s<R$ (we may choose with- 
out loss of generality $s>r)$ so that $\left\{y \in F: q_{s}(y) \leq b\right\} \subset T\left(\left\{x \in E: p_{r}(x) \leq 1\right\}\right)$ or equivalently, $p_{r}(x) \leq \frac{1}{b} q_{s}(T(x)) \forall x \in E$.

Now in a similar way as above we get the two remaining inequalities.

Corollary 10. Let $\left(E_{n},\|.\|_{n}\right)_{n}$ and $\left(F_{n},\|.\|_{n}\right)_{n}$ be $R$-Schauder and $R^{\prime}$ Schauder decompositions of $E$ and $F$ respectively $\left(0<R, R^{\prime}<\infty\right)$.

If there exists an algebraic isomorphism $T_{m}: E_{m} \longrightarrow F_{m}$ for every $m \in \mathrm{N}$ such that (Condition I') for each $t>1$ there exist $a_{t}, b_{t}>0$ so that

$$
\left\|T_{m}\left(x_{m}\right)\right\|_{m} \leq a_{t} t^{m}\left(\frac{R}{R^{\prime}}\right)^{m}\left\|x_{m}\right\|_{m} \text { and }\left\|x_{m}\right\|_{m} \leq b_{t} t^{m}\left(\frac{R^{\prime}}{R}\right)^{m}\left\|T_{m}\left(x_{m}\right)\right\|_{m},
$$

$\forall x_{m} \in E_{m}, \forall m \in \mathrm{N}$, then the map $x=\sum_{m=0}^{\infty} x_{m} \in E \longrightarrow T(x):=\sum_{m=0}^{\infty} T_{m}\left(x_{m}\right)$ $\in F$ is a topological isomorphism.

Conversely, if there exists a topological isomorphism $T: E \longrightarrow F$ such that $T_{m}\left(E_{m}\right) \subset F_{m} \quad \forall m \in \mathrm{N}$, then $T_{m}\left(E_{m}\right)=F_{m}$ and $T_{m}:=\left.T\right|_{E_{m}}$ are topological isomorphisms satisfying Condition I'.

Proof. It follows from Theorem 9.

Corollary 11. Let $\left(E_{n},\|\cdot\|_{n}\right)_{n}$ and $\left(F_{n},\|\cdot\|_{n}\right)_{n}$ be R-Schauder decompositions of $E$ and $F$ respectively $(0<R \leq \infty)$. If $E_{n}$ is isometrically isomorphic to $F_{n}$ for every $n \in \mathrm{N}$, then $E$ and $F$ are topologically isomorphic.

In the sequel we apply the above results to our motivating spaces.

Let $\mathcal{F}$ be a closed subspace of $\left(\mathcal{H}_{b}(U), \tau_{b}\right)$. The map $\delta$ given by $x \in U \longrightarrow \delta_{x} \in \mathcal{F}^{*}$, where $\delta_{x}(f)=f(x)$ for all $f \in \mathcal{F}$, is a holomorphic mapping of bounded type [12] and its adjoint map is $\delta^{*}: F \in \mathcal{F}^{* *} \longrightarrow F \circ \delta \in \mathcal{H}_{b}(U)$.

Definition 12. The space $\mathcal{F}^{* *}$ is said to be canonically isomorphic to a closed subspace $\mathcal{G}$ of $\left(\mathcal{H}_{b}(U), \tau_{b}\right)$ if $\delta^{*}: \mathcal{F}^{* *} \longrightarrow \mathcal{G}$ is a topological isomorphism.

If $\mathcal{F}=\mathcal{H}_{b}(U)$, then the map $\delta^{*}$ is defined between $\mathcal{H}_{b}(U)^{* *}$ and $\mathcal{H}_{b}(U)$. If we consider the space $\mathcal{H}_{b}(U)$ as a subspace of its bidual space by means of the natural injection, the map $\delta^{*}$ is a projection. Hence, if $\delta^{*}$ is also one-toone, the space $\mathcal{H}_{b}(U)$ is reflexive and $\delta^{*}$ is the identity map. In general, if $\mathcal{F}$ is a closed subspace of $\left(\mathcal{H}_{b}(U), \tau_{b}\right)$, such that $\delta^{*}$ is one-to-one and $\delta^{*}\left(\mathcal{F}^{* *}\right)=\mathcal{F}$, then the space $\mathcal{F}$ is reflexive.

We denote $\delta_{m}^{*}:=\left.\delta^{*}\right|_{\mathcal{F}_{m}^{* *}}$ where $\mathcal{F}_{m}:=\mathcal{F} \cap \mathcal{P}\left({ }^{m} X\right)$. Actually, $\delta_{m}^{*}$ is the adjoint map of the $m$-homogeneous polynomial $\delta_{m}: x \in X \longrightarrow \delta_{m, x} \in \mathcal{F}_{m}^{*}$, where $\delta_{m, x}\left(P_{m}\right)=P_{m}(x)$, so $\delta_{m}^{*}: \mathcal{F}_{m}^{* *} \longrightarrow \mathcal{P}\left({ }^{m} X\right)$. Also $\left\|\delta_{m}^{*}\right\| \leq 1 \quad \forall m \in \mathrm{N}$. If $\mathcal{F}_{m}=\mathcal{P}\left({ }^{m} X\right), \delta_{m}^{*}: \mathcal{P}\left({ }^{m} X\right)^{* *} \longrightarrow \mathcal{P}\left({ }^{m} X\right)$ is also a projection. Consequently, if $\delta_{m}^{*}$ is one-to-one then $\mathcal{P}\left({ }^{m} X\right)$ is reflexive and $\delta_{m}^{*}$ is the identity.

TheOREM 13. Let $X$ be a Banach space and let $U$ be either a bounded ba- 
lanced open subset of $X$ or $U=X$. Let $\mathcal{E}$ and $\mathcal{F}$ be closed subspaces of $\left(\mathcal{H}_{b}(U), \tau_{b}\right)$. Put $\mathcal{F}_{m}:=\mathcal{F} \cap \mathcal{P}\left({ }^{m} X\right)$ and $\mathcal{E}_{m}:=\mathcal{E} \cap \mathcal{P}\left({ }^{m} X\right)$ endowed with $\|.\|_{U}$ if $U$ is a bounded balanced open subset of $X$ and with $\|.\|_{B}$ if $U=X$. Assume that $\left(\mathcal{E}_{m}\right)_{m}$ and $\left(\mathcal{F}_{m}\right)_{m}$ are Schauder decompositions of $\mathcal{E}$ and $\mathcal{F}$ respectively. If $\mathcal{E}_{m}$ and $\mathcal{F}_{m}$ are (canonically) isometrically isomorphic, $\forall m \in \mathrm{N}$, then $\mathcal{E}$ and $\mathcal{F}$ are (canonically) topologically isomorphic.

Proof. It follows from Example 2, Remark 3 and Corollary 11.

Theorem 14. Let $X$ be a Banach space, $B$ the open unit ball of $X$ and $U$ either a bounded balanced open subset of $X$ or $U=X$. Let $\mathcal{F}$ be a closed subspace of $\left(\mathcal{H}_{b}(U), \tau_{b}\right)$. Let $\tau$ be a locally convex topology on $\mathcal{H}_{b}(U)$ weaker than or equal to $\tau_{b}$. Let $\mathcal{F}_{m}:=\left(\mathcal{F} \cap \mathcal{P}\left({ }^{m} X\right),\|.\|_{B}\right)$ if $U=X$ and $\mathcal{F}_{m}:=(\mathcal{F} \cap$ $\left.\mathcal{P}\left({ }^{m} X\right),\|.\|_{U}\right)$ if $U$ is a bounded balanced open subset of $X$. Assume

1) $\left(\mathcal{F}_{m}\right)_{m \in \mathrm{N}}$ is a Schauder decomposition of $\mathcal{F}$, and

2) If $f \in \overline{\mathcal{F}}^{\tau}$ then $P_{m}(f) \in \overline{\mathcal{F}}_{m}^{\tau}$ for every $m \in \mathrm{N}$ (equivalently, $\left(\overline{\mathcal{F}}_{m}^{\tau}\right)_{m}$ is a Schauder decomposition of $\overline{\mathcal{F}}^{\tau}$ ).

If there exist topological (canonical) isomorphisms $T_{m}: \mathcal{F}_{m}^{* *} \longrightarrow \overline{\mathcal{F}}_{m}{ }^{\tau} s a$ tisfying either Condition $I$ if $U$ is a bounded balanced open subset of $X$, or Condition II if $U=X, \forall m \in \mathrm{N}$, then $\mathcal{F}^{* *}$ is topologically (canonically) isomorphic to $\overline{\mathcal{F}}^{\tau}$.

Conversely, if there exists a topological isomorphism $T: \mathcal{F}^{* *} \longrightarrow \overline{\mathcal{F}}^{\tau}$ so that $T\left(\mathcal{F}_{m}^{* *}\right) \subset \overline{\mathcal{F}_{m}}{ }^{\tau}, \forall m \in \mathrm{N}$, then $T\left(\mathcal{F}_{m}^{* *}\right)=\overline{\mathcal{F}}_{m}{ }^{\tau}$ and $T_{m}:=\left.T\right|_{\mathcal{F}_{m}^{* *}}$ are topological isomorphisms satisfying Condition I if $U$ is a bounded balanced open subset of $X$, or Condition II if $U=X$.

Proof. Let $R$ be either $\infty$ if $U=X$ or 1 in other case. By Examples 2.a), 2.c) and Remark 3 the families $\left(\mathcal{F}_{m}\right)_{m}$ and $\left(\overline{\mathcal{F}}_{m}{ }^{\tau}\right)_{m}$ are $R$-Schauder decompositions of $\mathcal{F}$ and $\overline{\mathcal{F}}^{\tau}$ respectively. By Theorem 8 , the family $\left(\mathcal{F}_{m}^{* *}\right)_{m}$ is an $R$-Schauder decomposition of $\mathcal{F}^{* *}$. An application of Theorem 9 completes the proof.

COROLlary 15. Under the hypothesis of Theorem 14, if each $\mathcal{F}_{m}^{* *}$ is isometrically (canonically) isomorphic to $\overline{\mathcal{F}}_{m}{ }^{\tau}$, then $\mathcal{F}^{* *}$ is topologically (canonically) isomorphic to $\overline{\mathcal{F}}^{\tau}$.

We now see some applications of these results to the study of biduality of spaces of holomorphic functions.

Corollary 16. Let $X$ be a Banach space. Let $G \subset X^{*}$ be either the open unit ball of $X^{*}$ or $G=X^{*}$. If $\mathcal{P}_{w^{*}}\left({ }^{m} X^{*}\right)$ contains no copy of $\ell^{1}$ for every $m \in \mathrm{N}$, then $\mathcal{H}_{w^{*}}(G)^{* *}$ is canonically isomorphic to ${\overline{\mathcal{H}_{w^{*}}(G)}}^{\tau_{0}}$, the closure of $\mathcal{H}_{w^{*}}(G)$ in $\left(\mathcal{H}_{b}(G), \tau_{0}\right)$.

In particular, the isomorphism holds whenever $X$ is an Asplund space. 
Proof. To show that the conditions of Corollary 15 are fulfilled when $\mathcal{F}=\mathcal{H}_{w^{*}}(G)$ and $\tau=\tau_{0}$, we need to check that $P_{m} f \in{\overline{\mathcal{P}_{w^{*}}\left({ }^{m} X^{*}\right)}}^{\tau_{0}}$ whenever $f \in{\overline{\mathcal{H}_{w^{*}}(G)}}^{\tau_{0}}$. If $f \in{\overline{\mathcal{H}_{w^{*}}(G)}}^{\tau_{0}}$ there exists a net $\left(f_{i}\right)_{i \in I}$ in $\mathcal{H}_{w^{*}}(G)$ which $\tau_{0^{-}}$ converges to $f$. By the Cauchy inequalities, for each $m \in \mathrm{N}$, the net $\left(P_{m} f_{i}\right)_{i \in I} \subset \mathcal{P}_{w^{*}}\left({ }^{m} X^{*}\right) \tau_{0}$-converges to $P_{m} f$. Hence $P_{m} f \in{\overline{\mathcal{P}} w^{*}\left({ }^{m} X^{*}\right)}^{\tau_{0}}$.

Since $\mathcal{P}_{w^{*}}\left({ }^{m} X^{*}\right)$ contains no copy of $\ell^{1}, \mathcal{P}_{w^{*}}\left({ }^{m} X^{*}\right)^{* *}$ and $\overline{\mathcal{P}_{w^{*}}\left({ }^{m} X^{*}\right)}$ are canonically isometrically isomorphic for every $m \in \mathrm{N}$ (Theorem 2 of [14]). Corollary 15 now implies that $\mathcal{H}_{w^{*}}(G)^{* *}$ is canonically isomorphic to $\overline{\mathcal{H}}_{w^{*}}(G){ }^{\tau_{0}}$.

If $X$ is Asplund, $\mathcal{P}_{w^{*}}\left({ }^{m} X^{*}\right)$ is Asplund too (Corollary 1.1 of [14], see also the proof of Theorem 5 in [4]), $\forall m \in \mathrm{N}$. Hence, $\mathcal{P}_{w^{*}}\left({ }^{m} X^{*}\right)$ contains no copy of $\ell^{1}, \forall m \in \mathrm{N}$. The first part of the Corollary now completes the proof.

COROllary 17. Let $X$ be a Banach space such that $X^{*}$ has the approximation property. Let $G \subset X^{*}$ be either the open unit ball of $X^{*}$ or $G=X^{*}$. If $\left.\mathcal{P}_{w^{*}}{ }^{m} X^{*}\right)$ contains no copy of $\ell^{1}$ for every $m \in \mathrm{N}$, then $\mathcal{H}_{w^{*}}(G)^{* *}$ is canonically isomorphic to $\mathcal{H}_{b}(G)$.

In particular, the isomorphism holds whenever $X$ is an Asplund space such that $X^{*}$ has the approximation property.

Proof. Since $X^{*}$ has the approximation property, $\mathcal{P}_{w^{*}}\left({ }^{m} X^{*}\right)^{* *}$ and $\mathcal{P}\left({ }^{m} X^{*}\right)$ are canonically isometrically isomorphic (Theorem 3 of [14]). Examples 2.c), 4.c"), Theorem 8 and an application of Corollary 11 yield the result.

If $X$ is Asplund, $\left.\mathcal{P}_{w^{*}}{ }^{m} X^{*}\right)$ is Asplund too (Corollary 1.1 of [14]) for every $m \in \mathrm{N}$. Hence, for every $m \in \mathrm{N} \mathcal{P}_{w^{*}}\left({ }^{m} X^{*}\right)$ contains no copy of $\ell^{1}$. The first part of the Corollary completes the proof.

Corollaries 16 and 17 have been obtained by Valdivia in [15] for entire functions under the assumption that $\ell^{1}$ is not contained in the space of entire functions. J.C. Diaz pointed out to us that this assumption is equivalent to the non-containement of $\ell^{1}$ in $\left.\mathcal{P}_{w^{*}}{ }^{m} X^{*}\right) \forall m \in \mathrm{N}$ (Corollary 1.25 of [11]).

REMARK 18. Corollaries 16 and 17 hold for any open set $G$ in $X^{*}$ such that there exists a bounded balanced open subset $V$ of $X$ satisfying that $G$ coincides with the interior of $V^{\circ}$ for the norm topology in $X^{*}$. Indeed, let $\|\cdot\|_{V}$ be the Minkowski gauge of $V$ in $X$. The Banach space $Y:=\left(X,\|\cdot\|_{V}\right)$ is topologically isomorphic to $(X,\|\|$.$) and G$ is now the open unit ball of $Y^{*}$. Moreover, $\left.\mathcal{P}_{w^{*}}{ }^{*}\right)$ is isometrically equal to $\left(\mathcal{P}_{w^{*}}\left({ }^{m} X^{*}\right),\|\cdot\|_{G}\right)$ which clearly contains no copy of $\ell^{1}$. The conclusion follows from Corollary 16 (respectively Corollary 17).

Corollary 19. Let $X$ be a Banach space and let $U \subset X$ be either a boun- 
ded balanced open subset of $X$ or $U=X$. Assume that $\mathcal{P}_{w u}\left({ }^{m} X\right)$ contains no copy of $\ell^{1}$ for every $m \in \mathrm{N}$ (for example when $X^{*}$ is an Asplund space). Then

a) $\mathcal{H}_{w u}(U)^{* *}$ is topologically isomorphic to $\overline{\mathcal{H}_{w^{*}}\left(U^{* *}\right)^{\tau_{0}}}$, where $U^{* *}$ is the interior on $X^{* *}$ for the norm topology of the closure of $U$ for the weak*-topology on $X^{* *}$.

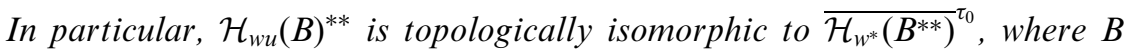
and $B^{* *}$ are the open unit balls of $X$ and $X^{* *}$ respectively, and $\mathcal{H}_{w u}(X)^{* *}$ is topologically isomorphic to $\overline{\mathcal{H}_{w^{*}}\left(X^{* *}\right)^{\tau_{0}}}$.

b) Moreover, if $X^{* *}$ has the approximation property then $\mathcal{H}_{w u}(U)^{* *}$ is topologically isomorphic to $\mathcal{H}_{b}\left(U^{* *}\right)$.

Proof. a) Since $\mathcal{P}_{w u}\left({ }^{m} X\right)$ and $\mathcal{P}_{w^{*}}\left({ }^{m} X^{* *}\right)$ are isometrically isomorphic, $\mathcal{P}_{w^{*}}\left({ }^{m} X^{* *}\right)$ contains no copy of $\ell^{1}, m \in \mathrm{N}$. Since the norm closure $\overline{U^{* *}}$ of $U^{* *}$ agrees with the weak*-closure of $U$, by the bipolar theorem $\overline{U^{* *}}$ is the bipolar of $U$ in $X^{* *}$. Thus, by Corollary 16 and Remark $18, \mathcal{H}_{w^{*}}\left(U^{* *}\right)^{* *}$ and $\overline{\mathcal{H}}_{w^{*}}\left(U^{* *}\right)^{\tau_{0}}$ are topologically isomorphic. Now, since $\mathcal{H}_{w u}(U)$ and $\mathcal{H}_{w^{*}}\left(U^{* *}\right)$ are topologically isomorphic, we finally obtain that $\mathcal{H}_{w u}(U)^{* *}$ and $\overline{\mathcal{H}_{w^{*}}\left(U^{* *}\right)} \tau_{0}$ are topologically isomorphic.

b) An analogous proof to the one in Corollary 17 gives the conclusion.

Corollary 20. Let $X$ be a Banach space and let $U \subset X$ be either the open unit ball of $X$ or $U=X$. If for every $m \in \mathrm{N} \mathcal{P}_{w u}\left({ }^{m} X\right)^{* *}$ is (canonically) isometrically isomorphic to $\mathcal{P}\left({ }^{m} X\right)$, then $\mathcal{H}_{\text {wu }}(U)^{* *}$ is (canonically) isomorphic to $\mathcal{H}_{b}(U)$.

Proof. Let $R$ be either $\infty$ if $U=X$ or 1 in other case. By Examples 2.a) and 2.c) $\left(\mathcal{P}\left({ }^{m} X\right)\right)_{m}$ is an $R$-Schauder decomposition of $\mathcal{H}_{b}(U)$. On the other hand, by Examples 4.a), 4.c) and Theorem $\left.8,\left(\mathcal{P}_{w u}{ }^{m} X\right)^{* *}\right)_{m}$ is an $R$-Schauder decomposition of $\mathcal{H}_{w u}(U)^{* *}$. Hence, Corollary 11 yields the result.

Corollary 20 and Corollary 21 below clarify Theorem 9 of [13].

Let us now consider the map $\widetilde{\delta}_{m}: z \in X^{* *} \longrightarrow \widetilde{\delta}_{m, z} \in \mathcal{P}\left({ }^{m} X\right)^{*}$, where $\widetilde{\delta}_{m, z}(P)=\widetilde{P}(z)$ and where $\widetilde{P}$ denotes the Aron-Berner extension [3] of $P$ to $X^{* *}$. González in [7] has defined, extending an earlier definition of Aron and Dineen [4], a Banach space $X$ to be Q-reflexive if the adjoint map $\widetilde{\delta}_{m}^{*}: \mathcal{P}\left({ }^{m} X\right)^{* *} \longrightarrow \mathcal{P}\left({ }^{m} X^{* *}\right)$ of $\widetilde{\delta}_{m}$ is bijective and hence, a topological isomorphism for every $m \in N$. Since $\left\|\widetilde{\delta}_{m}^{*}\right\| \leq 1$, in order to satisfy the converse inequalities in the hypothesis of Theorem 9 one has to assume $\tilde{\delta}_{m}^{*}$ to have some additional properties, for example to be isometries (in this case let us call $X$ to be isometrically $Q$-reflexive). So as a consequence of Corollary 11 we get the following result (compare with Proposition 16 of [4]).

Corollary 21. Let $X$ be an isometrically $Q$-reflexive Banach space and 
let $U \subset X$ be either the open unit ball of $X$ or $U=X$. Then the space $\mathcal{H}_{b}(U)^{* *}$ is topologically isomorphic to $\mathcal{H}_{b}\left(U^{* *}\right)$.

In fact, we can state the following theorem:

Theorem 22. Let $X$ be a Banach space and let $U \subset X$ be either the open unit ball of $X$ or $U=X$. Then the space $\mathcal{H}_{b}(U)^{* *}$ is topologically isomorphic to $\mathcal{H}_{b}\left(U^{* *}\right)$ if, and only if, $X$ is $Q$-reflexive and the sequence $\left(\tilde{\delta_{m}}\right)_{m}$ satisfies either Condition I if $U \neq X$ or Condition II if $U=X$.

Acknowledgement. Thanks are given to Domingo García for the helpful discussions we had with him during the preparation of this paper.

\section{REFERENCES}

1. J. M. Ansemil, S. Ponte, An example of quasinormable Fréchet function space which is not a Schwartz space, S. Machado (ed.), Functional analysis, holomorphy and approximation theory, 1-8, Lecture Notes in Math. 843 (1981).

2. R. Aron, Weakly uniformly continuous and weakly sequentially continuous entire functions, J. A. Barroso (ed.), Advances in holomorphy, 47-66. Notas Mat. 65 Amsterdam. NorthHolland (1979).

3. R. Aron, P. Berner, A Hahn-Banach extension theorem for analytic mappings, Bull. Soc. Math. France 106 (1978), 3-24.

4. R. Aron, S. Dineen, Q-reflexive Banach spaces, Rocky Mountain J. Math. 27 (1997), 10091025.

5. K.D. Bierstedt, R.G. Meise, W.H. Summers, Köthe sets and Köthe sequence spaces, Functional Analysis, Holomorphy and Approximation Theory, J.A. Barroso (Ed.), Amsterdam-London (1982), 27-91.

6. S. Dineen, Complex analysis in locally convex spaces, North-Holland Math. Studies, Vol. 57, North-Holland, Amsterdam, 1981.

7. M. González, Remarks on Q-reflexive Banach spaces, Proc. Roy. Irish Acad. 96 (1996), 195201.

8. J. Jaramillo, A. Prieto and I. Zalduendo, The bidual of the space of polynomials on a Banach space, Math. Proc. Cambridge Philos. Soc. 122 (1997), 457-471.

9. H. Jarchow, Locally convex spaces, B. G. Teubner Stuttgart, 1981.

10. G. Köthe, Topological Vector Spaces, Springer (1969- 79).

11. M.A. Miñarro, Descomposiciones de espacios de Fréchet. Aplicación al producto tensorial proyectivo, Tesis Doctoral (1991).

12. J. Mujica, Linearization of holomorphic mappings of bounded type, Progress in Functional Analysis, North-Holland Math. Stud. 170 (1992), 149-162.

13. A. Prieto, The bidual of spaces of holomorphic functions in infinitely many variables, Proc. Roy. Irish Acad. Sect. A 92 (1992), 1-8.

14. M. Valdivia, Banach spaces of polynomials without copies of $\ell^{1}$, Proc. Amer. Math. Soc. 123 (1995), 3143-3150.

15. M. Valdivia, Fréchet spaces of holomorphic functions without copies of $\ell^{1}$, Math. Nachr. 181 (1996), 277-287.

DEPARTAMENTO DE ANÁLISIS MATEMÁTICO

UNIVERSIDAD DE VALENCIA

E-46100 BURJASSOT (VALENCIA) 